\title{
A Study on the Construct Validity of the Brief Fear of Negative Evaluation Scale (BFNE)
}

\author{
Sabahattin Çam, PhD \\ Esef Ercüment Yerlikaya, PhD \\ Çukurova University, Adana, Turkey
}

Doi:10.19044/ejes.v7no4a4 URL:http://dx.doi.org/10.19044/ejes.v7no4a4

\begin{abstract}
This study aimed to test the construct validity of three different forms of the Fear of Negative Evaluation Scale. The Brief Fear of Negative Evaluation Scale (BFNE), the Brief Fear of Negative Evaluation ScaleRevised (BFNE-II) and the Brief Fear of Negative Evaluation ScaleStraightforward (BFNE-S) were applied to a group of 652 people in total, including 339 females, 313 males, 320 university students, and 332 high school students. To examine the factor structure of the scales, confirmatory factor analysis was performed. Item analysis were conducted to compare the psychometric properties of straightforwardly scored and reverse-scored items. To investigate the internal consistency coefficients of the scales, Cronbach's alpha coefficients were calculated. As a result of the confirmatory factor analysis performed for three different forms of the scale, it was found that the BFNE did not fit well to a two-factor model, while the BFNE-II and BFNE-S fit well to a single-factor model. The item analyses conducted for three different forms, and the calculated internal consistency coefficients also revealed that the BFNE-II and BFNE-S had better psychometric properties than the BFNE. These findings are in parallel with the findings obtained in studies conducted on the construct validity of the original English version of the BFNE in the last 15 years. Based on these findings, it was concluded that BFNE-S is the appropriate tool to measure the fear of negative evaluation of high school and university students in Turkey due to its theoretically based, robust factor structure and its high internal consistency coefficient despite consisting of fewer items.
\end{abstract}

Keywords: BFNE, BFNE-II, BFNE-S, validity, reliability. 


\section{Introduction}

The Brief Fear of Negative Evaluation Scale (BFNE; Leary, 1983) is a tool that aims to measure a dimension of social anxiety (Leary, 1983). The scale consists of 12 items with answer options between "not at all characteristic of me" (1) and "extremely characteristic of me" (5). Eight of the items are straightforwardly scored, and four items are reverse-scored. Leary (1983) revealed that the brief version of the scale had quite similar psychometric properties to the original version, developed by Watson and Friend (1969) and consisting of 30 items. Since the years when the BFNE was revised, it has been stated to be the most widely used tool to measure the fear of negative evaluation of individuals (Carleton, Collimore, McCabe \& Anthony, 2011). However, despite its common use, many studies have been carried out on the construct validity of the scale. Not only this but also discussions based on the results of these studies have continued for many years.

The scale was assumed to have a single-factor structure until the study examining the construct validity of the long and brief versions of the Fear of Negative Evaluation Scale conducted by Rodebaugh, Woods, Thissen, Heimberg, Chambless, and Rapee (2004) (Leary, 1983; Turner, McCanna \& Beidel, 1987; Stopa \& Clark, 2001). One of the two actual results reached by Rodebaugh et al. (2004) in this study is that the response approach based on five-point rating, instead of the double (dichotomous) response used in the extended version, allows for more sensitive measurement. The second result is that the single-factor structure assumed in previous studies, both in the short and long versions, was not confirmed. As a result of the confirmatory factor analysis (CFA), it was found that the single-factor structure had weak fit indices. The researchers tested the two-factor model by collecting the reversescored items and straightforwardly scored items of the brief version of the scale under different factors and allowing a correlation between these two factors. As a result of the test, it was found that the two-factor model had acceptable fit indices. In the study comparing the two-factor and single-factor models related to the scale, it was determined that the two-factor model showed a significantly better fit than the single-factor model. In the study, it was stated that the two-factor model had better fit indices, but the reverseworded items could cause confusion and erroneous responses in respondents. To avoid this situation, it was recommended that only straightforwardly worded items should be included in the brief version of the scale. Thus, it was suggested that both the reliability and the discriminant validity of the scale would increase.

In another similar study on the scale (Weeks, Heimberg, Fresco, Hart, Turk, Schneier \& Liebowitz, 2005), it was concluded that the BFNE had a two-factor structure consisting of straightforwardly worded and reverse- 
worded items. In this study, it was observed that the researchers, who reached a conclusion parallel to the result obtained by Rodebaugh et al. (2004) regarding the construct validity of the scale, also agreed with the concerns of Rodebaugh et al. (2004) about using the scale with both straightforwardly and reverse-worded items.

From the findings obtained in these two studies, it is found that the straightforwardly worded items of the scale provide a psychometrically, more robust measure of the fear of negative evaluation, that is highly correlated with other scales measuring social anxiety and is more sensitive in detecting intervention-based changes in fear of negative evaluation. Furthermore, it is emphasized that the reverse-scored items scored in the past uses of the scale may have caused errors in the measurement of the fear of negative evaluation. Since the scale is widely used both in practice and research, it is observed that the researchers (Rodebaugh et al., 2004; Weeks et al., 2005) suggest revising the scale based on the results they reached.

Carleton, McCreary, Norton, and Asmundson (2006), who evaluated the results of the studies conducted to date on the scale and the criticism and suggestions brought to the construct validity of the scale, carried out a study to repeat the results of the factor analysis previously conducted (Rodebaugh et al., 2004; Weeks et al., 2005) and to determine the most appropriate way to be followed for reverse-worded items. Three possible options were discussed in the study: (1) to include reverse-scored items in the scale, but not include them in the scoring, (2) to remove these items from the scale, and (3) to word these items straightforwardly. As a result of the CFA performed for the original BFNE with four reverse-scored items and the BFNE-II with these items revised to be straightforwardly worded, it was found that the two-factor structure for the BFNE fit the data better. In contrast, the single-factor structure for the BFNE-II fit the data better.

Furthermore, while the Cronbach's alpha internal consistency coefficient was calculated to be .89 for the BFNE, this value was calculated to be .95 for the BFNE-II. Based on these results, Carleton et al. (2006) suggested the use of the BFNE-II, instead of removing the reverse-scored items from the scale or leaving them in the scale and not including in the scoring. (It is also observed that this version of the scale is named the BFNE-R in some publications).

Shortly after this study, Carleton, Collimore, and Asmundson (2007) created a shorter 8-item version of the BFNE-II (BFNE-R) in their research and compared the psychometric properties of the 12-item version and the 8item version. When creating the eight-item version, the researchers used seven of the straightforwardly scored items in the original scale and one of the four items revised to be straightforwardly worded. As a result of their analysis, Carleton et al. (2007) stated that the 8-item version had stronger psychometric 
properties compared to the 12-item version and suggested using the 8-item version.

In the studies conducted on the BFNE by different research groups, with additional samples or using various analyses (Rodebaugh et al., 2004; Weeks et al., 2005; Collins, Westra, Dozois \& Stewart, 2005; Duke, Krishnan, Faith \& Storch, 2006; Carleton, McCreary, Norton \& Asmundson, 2006; Carleton, Collimore \& Asmundson, 2007; Rodebaugh, Woods \& Heimberg, 2007), similar results are observed. In these studies, it is stated that the straightforwardly worded BFNE items constituted a single-factor structure with sufficient convergent and divergent validity. The reverse-scored items, in contrast, constituted only a methodology-based factor without a theoretical basis, and this could destabilize the findings obtained.

The purpose of including reverse-scored items in scales is to make more appropriate measurements, especially in attitude scales, and to detect inconsistent responses in long scales with many items. Although this application is considered necessary for the original 30-item form of the scale (Watson \& Friend; 1969), it may be regarded as not necessary for the 12-item brief version (BFNE). Furthermore, many studies have shown that the items in question constitute a separate factor that does not have a theoretical basis due to their way of expression. For these reasons, the research groups examining the psychometric properties of the BFNE revealed the following three possible options for the revision and use of the scale items.

One of these options is to use only eight straightforwardly worded items on the scale (Rodebaugh et al., 2004; Weeks et al., 2005). The form consisting of these eight items was named the BFNE-S to emphasize that it consists of straightforwardly scored items. Another option is to revise the four reverse-worded items to be straightforwardly worded and use the scale as 12 items (Carleton, McCreary, Norton \& Asmundson, 2006; Taylor, 1993). This form, consisting of 12 straightforwardly worded items, was named the BFNEII (BFNE-R) to emphasize that it was revised. The third option is to use a shorter eight-item version of the BFNE-II, which contains seven of the items included in the revised 12-item scale (BFNE-II) and straightforwardly worded in the original scale and one of the items revised to be straightforwardly worded (Carleton, Collimore \& Asmundson, 2007).

In their study conducted to compare these three different options, Carleton, Collimore, McCabe, and Anthony (2011) revealed that the three different forms of the tool mentioned above exhibited a single-factor structure. Moreover, it was observed that the BFNE-S and BFNE-II (BFNE-R) forms of the scale had better fit indices for a single-factor structure compared to the eight-item short version of the BFNE-II. Another critical finding reached in the study is that the BFNE-S, which consists of eight items straightforwardly worded in the original scale, is more successful than the other forms of the 
scale in predicting social anxiety in the clinical sample. Based on these findings, Carleton et al. (2011) suggest that the reverse-worded BFNE items, which they consider to be unnecessary and potentially problematic, should be removed from the scale and that the use of the BFNE-S will be more appropriate both in research and for clinical purposes. Likewise, as a result of their study comparing the factor structures of the different forms of the scale, Liu and Love (2016) revealed that the BFNE-S (8 items) fit the single-factor structure better than the BFNE-II (12 items).

It is observed that three different research groups carried out the adaptation studies of the BFNE to Turkish culture. The first one of these is the study conducted by Koydemir and Demir (2007) with university students. In this study, the factor structure of the tool was examined as a two-factor structure, as was examined by Rodebaugh et al. (2004) and Weeks et al. (2005). As a result, the eight straightforwardly scored items were collected in a factor with the load values between .53 and .82 , and the four reverse-scored items were collected in the second factor with the load values between .46 and .84. In the criterion validity study, sufficient evidence was obtained for the total score and the scores obtained from the two factors. It was found that the internal consistency coefficients of the factor scores and the total score were also high.

The second adaptation study of the BFNE to Turkish was performed by Bilge and Kelecioğlu (2008) on high school students by translating the scale items into Turkish again. Although the study mentioned the existence of research conducted by converting the expressions of reverse-scored items into straightforward, it was observed that the two-factor structure was tested while examining the construct validity of the tool. In this study, it was concluded that the Turkish form of the scale consisted of a two-factor structure with a total of 11 items, as it was observed that item 4 was not loaded on the relevant factor with an acceptable value. Eight straightforwardly scored items were placed in the first factor, and three reverse-scored items were placed in the second factor. While sufficient evidence was obtained for the first factor in the examination of criterion validity, it was observed that these pieces of evidence were insufficient for the second factor, which included three items. Moreover, considering both internal consistency and test-retest reliability, it was observed that the reliability of the second factor was quite insufficient.

The third adaptation study of the BFNE was carried out by Çetin, Doğan, and Sapmaz (2010) with university students, by translating the scale items into Turkish once again. In this study, it was observed that the corrected item-total correlation value of the 4 th item was not sufficient $(r=-.03)$ and this item was not included in the analysis, and the psychometric properties of the scale were examined with 11 items. In the study, the construct validity of the tool was tested in terms of both single-factor and two-factor models. 
Considering the fit indices obtained, it was observed that both models fit the data well. Based on these results, the researchers took into account the previous single-factor model proposal of Collins et al. (2005). They concluded that the remaining reverse-scored three items on the scale did not measure a different structure from the other eight items. From this point of view, the criterion validity of the single-factor structure of the scale was examined, and as a result, significant pieces of evidence were obtained. It was observed that the scale, also in this form, had high internal consistency (.84), test split (.83), and test-retest reliability (.82).

When the results of the research in the international literature on the scale presented above are examined, it is observed that using only eight straightforwardly worded items will increase the validity and reliability of the measurements regarding the use of the BFNE. In the studies conducted on the fear of negative evaluation in the last decade (Weeks \& Howell, 2012; Levinson, Rodebaugh, White, Menatti, Weeks, Iacovino \& Warren, 2013; Le Blanc, Bruce, Heimberg, Hope, Blanco, Schneier \& Liebowitz, 2014; Menatti, DeBoer, Weeks \& Heimberg, 2015; Yap, Gibbs, Francis \& Schuster, 2016; Willarosa-Hurlocker, Whitley, Capron \& Madson, 2018; Sedighimornani, Rimes, Verplanken \& Gauntlett-Gilbert, 2019), it is observed that mostly the BFNE-S form of the scale is used. It is observed that the results obtained on the construct validity in the adaptation studies of the BFNE to Turkish are not consistent with each other, and the last two studies suggested using the scale with 11 items, in other words, with the number of items that is different from the original. In the studies examining the fear of negative evaluation, it can be said that using these different forms of the BFNE, which have different translation texts and different item numbers, may destabilize the measurement of the structure desired to be measured. When the use of the tool in the research in Turkey is examined in the last ten years, it is observed that all the three translation texts of the tool are used with the different number of the items (Doğan \& Totan, 2010; Karademir, 2011; Seçer, Halmatov \& Gençdoğan, 2013; Ömür, Aydın \& Argon, 2014; Irmak, 2015; Çetinkaya-Yıldız \& Toprak, 2016; Ben, 2017; Ümmet, Çağlar, İme \& Akyıl, 2018). This situation will prevent the comparison of national study results with each other and drawing general conclusions, as well as making it difficult to compare these results with the results of studies conducted in different cultures. It is considered important to carry out studies conducted on the construct validity of the original English version of the scale in the last 15 years for the Turkish version as well and to determine a standard form to be used in studies on the fear of negative evaluation. Therefore, it is necessary to examine the factor structures of different forms of the tool in Turkish culture and to compare their results. The results to be obtained in the study can eliminate measurement errors that will be created by using the scale with different texts and different item 
numbers in Turkey and will ensure the comparison of the results of studies to be conducted on the fear of negative evaluation with both national and international study results. Therefore, in this study, it was aimed to examine the factor structures of the original version of the BFNE (12 items), the revised form BFNE-II (12 items) created by revising the four reverse-scored items to be straightforwardly worded, and the form consisting of eight items scored straightforwardly in the original form (BFNE-S) in high school and university students.

\section{Method \\ Participants}

The research was conducted with a study group of 652 people in total, consisting of 320 university students and 332 high school students, receiving education in a state university and three public high schools that provide academic education in a city in the Eastern Mediterranean Region of Turkey. Both groups included high school students studying at all grade levels of three high schools and university students from all grade levels studying in six different programs. While collecting the data, groups were formed according to the data collection form by giving the BFNE form to one student and the BFNE-II form to a student sitting next to him/her, according to the sitting status of both high school and university students. Detailed information on the study groups is provided below.

The BFNE Applied Group: This group included 324 participants, 173 of whom were female and 151 were male. Of them, 164 were high school students $(55.5 \%$ female, $44.5 \%$ male), and 160 were university students (51.3\% female, $48.8 \%$ male). The age of high school students varied between 15 and 19 years, and the mean age was 16.79 years. The age of university students varied between 18 and 28 years, and the mean age was 21.41 years. There was no significant difference according to the education level and gender distribution of the participants $\left(\mathrm{X}^{2}=0.58 ; \mathrm{p}>0.05\right)$.

The BFNE-II Applied Group: In the second group in which the study was conducted, there were a total of 328 students, including 166 females and 162 males. Of them, 168 were high school students ( $53.0 \%$ female, $47 \%$ male), and 160 were university students (48.1\% female, $51.9 \%$ male). The age of the high school students in this group varied between 15 and 19 years, and the mean age was 16.80 years. It is observed that the age of university students varied between 18 and 28 years, and the mean age was 21.41 years, as in the other group. There was no significant difference according to the education level and gender distribution of the participants in this group $\left(X^{2}=0.77\right.$; $\mathrm{p}>0.05)$.

The BFNE-S Applied Group: Since the BFNE-S consists of eight straightforwardly worded items in both the BFNE and BFNE-II, the analysis 
for the BFNE-S was carried out on a group of 652 people in total, including 320 university students and 332 high school students in the two groups described above.

\section{Measures}

In this study, the Turkish form of BFNE, which was previously translated into Turkish by Koydemir and Demir (2007) and examined for its validity and reliability in a sample of Turkish university students was used.

BFNE: The scale includes a total of 12 items, eight of which are straightforwardly scored and four of which are reverse-scored. The items have five-point response options ( $1=$ Not at all characteristic of me and 5= Extremely characteristic of me). In the adaptation study of the BFNE performed with 250 university students, it was concluded that the scale exhibited a two-factor structure. Eight straightforwardly scored items were loaded with appropriate values in the first factor, and the four reverse-scored items were loaded with appropriate values in the second factor. The correlation value of the first factor with the total score was .97 , of the second factor with the total score was .90 , and the correlation value between the two factors was .76. Significant relationships were found between the Revised Cheek and Buss Shyness Scale score and the BFNE total score at a value of .33, with a score obtained from eight items at a value of .34, and with a score of four items at a value of .27. The correlation values calculated for the relationships with the Rosenberg Self-Esteem Scale scores are -.21, -.22, and -.17, respectively. The Cronbach's alpha internal consistency coefficients were calculated to be .94 for the total score, .91 for eight items, and .87 for four items. The item-total score correlation values also vary between .61 and .78 (Koydemir \& Demir, 2007).

BFNE-II: In this version of the scale, the 2nd, 4th, 7th and 10th items, which were reverse-scored within the framework of the explanations made in the introduction section of the study, were straightforwardly worded, by allowing the scoring as presented below.

Item 2: Even if I do not know for sure that people have a bad impression of me, I become obsessed with this.

Item 4: I am very concerned about what kind of impression I make on someone.

Item 7: The opinions of others about me bother me.

Item 10: Knowing that someone is judging me influences me a lot. 


\section{Data Collection and Analysis}

For the data collection process, verbal permissions were obtained from the school administration in high schools and from the lecturers of the students at the university. The branches/groups determined to be suitable at the time of data collection were identified in both groups. Information about the purpose and application process of the study was provided to these groups by entering the classrooms at the beginning of the lesson. Besides, scales were distributed to those who wanted to answer the measurement tool voluntarily. One of the students sitting close to each other in each classroom was given the BFNE randomly, and the student next to him/her was given the BFNE-II. It took approximately 10 minutes to fill out the scales in all groups.

The collected data were transferred to the SPSS 22.0 program, and the descriptive analysis of the items was performed. For item analysis, the correlations of the items in the scale with the total score were examined. The internal consistency reliability was calculated using Cronbach's alpha method. Furthermore, whether the distribution of the students in the two study groups differed according to gender and grade level was examined by the $\mathrm{X}^{2}$ Independence Test. To examine the construct validity of the scale, confirmatory factor analysis (CFA) was conducted using the IBM AMOS 22 statistics program. The significance level of 0.05 was taken as a criterion in the interpretation of the results.

\section{Results}

\section{Item Descriptive Values and Item Analysis}

In the group in which the BFNE was applied $(\mathrm{N}=324)$, it was observed that the mean values of the items in the data set varied between 2.34 and 3.67 and the standard deviation values varied between 1.16 and 1.36. The skewness coefficients varied between -0.62 and 0.61 , and the kurtosis coefficients varied between -0.40 and -1.18 . A striking finding in this data set is that the mean scores of eight straightforwardly scored items varied between 2.34 and 2.80, while the values of four reverse-scored items varied between 3.27 and 3.67. As can be seen, each of these four items increases the total score by approximately one point compared to the other items. In this structure in which the scale is assumed to have two factors, the item-total score correlation values with the items in the factor containing eight items varied between .54 and .67 $(p<.05)$, while one of the four reverse-scored items (4th item, $r=.02 ; p>0.05$ ) did not show a significant relationship with the total score, the correlation values of the other items varied between .27 and .34 ( $\mathrm{p}<.05)$. When the itemtotal score correlation values of a total of 12 items are examined, it is observed that the values of the eight straightforwardly worded items vary between .51 and .64 ( $\mathrm{p}<.05)$. However, contrary to what has been expected, it is observed that item 4 among the reverse-scored items has a correlation coefficient of - 
.22 , items 2 and 10 have a correlation coefficient of .19, and item 7 has a correlation coefficient of .28. Moreover, no significant relationship was found between the total scores obtained from eight items and four items ( $r=.06$; $\mathrm{p}>0.05)$. While the total score of eight items shows a correlation value of .93 with the score obtained from the overall scale, this value for four items is .43 $(\mathrm{p}<.05)$.

In the group in which the BFNE-II was applied $(\mathrm{N}=328)$, the mean scores of the items varied between 2.15 and 2.84, and the standard deviation values were found to vary between 1.11 and 1.36. It was observed that the skewness coefficients of the item scores varied between 0.24 and 0.82 , and the kurtosis coefficient values varied between -0.11 and -1.15 . In this data set, the mean values of the items straightforwardly worded in the BFNE varied between 2.15 and 2.84. Four items revised to be straightforwardly worded had average values between 2.25 and 2.77 , similar to the other items. The correlation values calculated with the total score of the 12 items in this form vary between .49 and $.74(\mathrm{p}<.05)$. A correlation value of .98 was obtained between the total score and the score obtained from eight items, and a correlation value of .90 was obtained between the score obtained from four items. In comparison, a correlation value of .78 was calculated between the scores obtained from eight and four items $(\mathrm{p}<.05)$.

When the data in the total sample of the BFNE-S $(\mathrm{N}=652)$ are examined, it is observed that the mean values of the items are between 2.51 and 2.82, and the standard deviation values are between 1.18 and 1.36. Furthermore, while the skewness values vary between 0.20 and 0.71 , the kurtosis values vary between -0.45 and -1.16 . The item-total score correlation values are between .56 and $.69(\mathrm{p}<.05)$.

\section{Construct Validity}

Confirmatory factor analysis was carried out to test the construct validity of the BFNE, BFNE-II, and BFNE-S forms, and in the evaluation of the model fit, the fit indices presented in Table 1 were calculated in line with the suggestions of Hu and Bentler (1999). 
Table 1: CFA model indices of BFNE, BFNE-II and BFNE-S

\begin{tabular}{lllllllll}
\hline & $\mathrm{X}^{2}$ & $\mathrm{p}$ & $\mathrm{X}^{2} / \mathrm{df}$ & $\mathrm{CFI}$ & $\mathrm{NFI}$ & $\mathrm{TLI}$ & $\begin{array}{l}\text { RMSE } \\
\mathrm{A}\end{array}$ & SRMR \\
\hline BFNE & 154, & .00 & 2,91 & .91 & .87 & .88 & .077 & .074 \\
& 05 & 0 & & & & & & \\
\hline BFNE-II & 172, & .00 & 3.20 & .93 & .90 & .91 & .082 & .045 \\
& 79 & 0 & & & & & & \\
\hline BFNE-S & 136, & 000 & 6.85 & .94 & .93 & .92 & .095 & .041 \\
& 93 & & & & & & & \\
\hline
\end{tabular}

BFNE: Brief Fear of Negative Evaluation Scale, BFNE-II: Brief Fear of

Negative Evaluation Scale-Revised, BFNE-S: Brief Fear of Negative Evaluation Scale Straightforward Items

As can be understood from the values in Table 1, although the CFI, RMSEA, and SRMR values met the acceptable fit criteria of the model in the BFNE form in which the two-factor structure of the scale was tested, it was found that the data in this model did not fit well to the model (NFI and TLI values <.90). Considering the fit indices obtained as a result of the confirmatory factor analysis in which the single-factor structure of the BFNEII, consisting of 12 items among which there are items revised to be straightforwardly worded, and the BFNE-S, which consists of only eight straightforwardly-scored items, was tested, it was concluded that the models showed an acceptable fit to the data for both forms.

The factor loadings, error variances, and t-values of the items related to the three forms of the tool as a result of the CFA are presented in Table 2. 
Table 2: Factor loadings, error variances and t-values of the items of BFNE, BFNE-II and BFNE-S

\begin{tabular}{llllllllll}
\hline \multicolumn{3}{c}{ BFNE } & \multicolumn{3}{c}{ BFNE-II } & \multicolumn{3}{c}{ BFNE-S } \\
\hline Item & $\mathrm{R}^{2}$ & $\mathrm{EV}$ & $\mathrm{t}$ & $\mathrm{R}^{2}$ & $\mathrm{EV}$ & $\mathrm{t}$ & $\mathrm{R}^{2}$ & $\mathrm{EV}$ & $\mathrm{t}$ \\
\hline 1 &, 61 & 1,02 & $11,22^{*}$ &, 71 & 1,14 & $12,02^{*}$ &, 64 & 1,07 & $16,19^{*}$ \\
3 &, 57 &, 67 & $10,70^{*}$ &, 62 & 1,11 & $12,34^{*}$ &, 60 &, 95 & $16,12^{*}$ \\
5 &, 67 &, 81 & $10,59^{*}$ &, 69 &, 71 & $11,41^{*}$ &, 69 &, 61 & $14,79^{*}$ \\
6 &, 73 &, 91 & $11,16^{*}$ &, 70 &, 89 & $12,21^{*}$ &, 73 &, 73 & $15,30^{*}$ \\
8 &, 72 &, 91 & $11,62^{*}$ &, 72 &, 65 & $11,65^{*}$ &, 72 &, 75 & $15,07^{*}$ \\
9 &, 72 & 1,12 & $10,60^{*}$ &, 79 &, 67 & $11,33^{*}$ &, 75 &, 78 & $15,75^{*}$ \\
11 &, 68 & 1,34 & $12,66^{*}$ &, 66 &, 87 & $11,91^{*}$ &, 66 &, 95 & $16,62^{*}$ \\
12 &, 67 & 1,18 & $9,12^{*}$ &, 62 &, 70 & $11,54^{*}$ &, 65 &, 84 & $16,26^{*}$ \\
2 &, 49 & .88 & $11,10^{*}$ &, 64 &, 97 & $11,84^{*}$ & & & \\
4 &,- 07 & .74 & $10,73^{*}$ &, 73 &, 52 & $10,74^{*}$ & & & \\
7 &, 68 & 1,00 & $11,81^{*}$ &, 56 &, 76 & $11,59 *$ & & & \\
10 &, 42 &, 98 & $4,74 *$ &, 51 &, 91 & $12,09^{*}$ & & & \\
\hline & & & & & $* \mathrm{p}<.05$ & & & &
\end{tabular}

BFNE: Brief Fear of Negative Evaluation Scale, BFNE-II: Brief Fear of

Negative Evaluation Scale-Revised, BFNE-S: Brief Fear of Negative Evaluation Scale Straightforward Items

When the values in Table 2 are examined, it is observed that the factor loadings of the eight items in the BFNE form, in which the two-factor structure of the tool was tested, were within acceptable limits. It was found that the fourth item, which is one of the reverse-scored items, had a negative factor load, contrary to what had been expected in the model. The correlation value between the two factors is .29 $(\mathrm{t}=2.96 ; \mathrm{p}<.05)$. In the BFNE-II, the factor loading values of 12 items varied between .51 and .79. In the BFNE-S form, the values of eight items were found to vary between .64 and .75. It is observed that the t-values calculated for the items in all three forms of the scale are significant at the level of 0.05 .

\section{Reliability}

To examine the reliability of the measurements, Cronbach's alpha internal consistency coefficients were calculated for three forms. As a result of the analysis, the values of .87 were obtained for the eight straightforwardly scored items of the BFNE, .42 for the four reverse-scored items, and .77 for the overall scale. The coefficient values for the BFNE-II form (with 12 items) and the BFNE-S form (with eight items) were respectively .90, and .87. 


\section{Discussion}

As stated above, the construct validity of the BFNE (12 items, including eight straightforwardly scored items and four reverse-scored items), BFNE-II (12 items, including eight straightforwardly scored items and four items revised to be straightforwardly worded), and the BFNE-S (eight items worded straightforwardly in the original scale) forms of the Fear of Negative Evaluation Scale was examined in high school and university students in the study. As a result of the CFA conducted, the fit index values obtained for the BFNE form and the factor loadings of the items showed that the tool was not suitable for the two-factor structure. This finding does not support the results of the adaptation studies to Turkish performed in university students (Koydemir \& Demir, 2007) and high school students (Bilge \& Kelecioğlu, 2008) in which one item (item 4) was excluded from the scale, and indicating that the tool has a two-factor structure. For the BFNE-II form in which the single-factor structure of the 12-item tool was tested, sufficient fit values of the scale to the single-factor structure were achieved. This result is in parallel with the opinion that it is appropriate to use the tool as a single-factor structure. However, the two-factor and single-factor structures of the tool were confirmed in another adaptation study to Turkish conducted with university students (Çetin, Doğan \& Sapmaz, 2010). Likewise, this finding of the research supports the findings of Collins et al. (2005) and Carleton et al. (2011), indicating that the single-factor structure has better fit index values. This finding can be considered as evidence for the views of many authors stating that the four reverse-scored items in the scale do not reflect a separate structure. Still, they come together merely because of the way the items are expressed. As a result of the CFA obtained for the BFNE-S form, which contains eight items straightforwardly worded in the original version of the tool, it was found that the model fit the data better. This finding supports the results and suggestions in the latest studies in the international literature on the validity of the scale (Carleton et al., 2011; Liu \& Love, 2016) that more valid and reliable measurements will be made with only these eight items.

Carleton et al. (2011) suggested that the tool was more successful in predicting social anxiety in the clinical sample with these eight items and that potentially problematic items should be removed, and the tool should be used both in the clinical setting and in research. When the descriptive values of the scale items were examined in the study, it was observed that each of the four reverse-worded items in the BFNE form had an item mean approximately one point higher than the other eight items. In the BFNE-II form, in which the statements of these items were revised to be straightforwardly worded, the mean item score values were found to be quite similar to the values of the other eight items. The reverse-scored items in the BFNE not only create a factor that 
does not have a theoretical basis by gathering under a different factor but also can cause erroneous decisions in cases when decisions are made, especially considering the scores obtained from the scale, such as making a diagnosis. The presence of reverse-worded items may cause the total scale score to increase by approximately four points.

In the item analysis conducted in the BFNE form, it was observed that item 4 did not have an item-total score correlation value in the expected direction, and the values of the other three items were lower in comparison with the straightforwardly scored eight items. This result can be interpreted as that the inclusion of the said items in the scale will weaken the validity and reliability of the measurements. While there was no significant relationship between the total scores of the four items and the eight items in the BFNE form $(r=.06)$, the scores obtained from the four reverse-scored items were moderately correlated with the total score of the scale $(\mathrm{r}=.43)$. There is a very high correlation between the eight-item scores and the total score $(r=.93)$. However, in the BFNE-II form, which included four items revised to be straightforwardly worded, a high level of positive correlation was observed between the scores of these items and the scores of eight items ( $r=.78)$ and the total score $(\mathrm{r}=.90)$. Likewise, there is a near-perfect $(\mathrm{r}=.98)$ correlation between the eight-item scores and the total score. These findings suggest that the four reverse-worded items do not have the desired psychometric properties in measuring the construct wanted to be measured, as well as they cause errors in measurements. It is regarded that the use of these items by revising them to be straightforwardly worded is more appropriate for the intended measurement. However, the considerably high correlation between the eight straightforwardly scored items and the total score in both forms (BFNE and BFNE-II) eliminates the need to use these reverse-scored items. This result is also supported by the results of the reliability analysis conducted for all three forms. The weakest reliability coefficient was calculated for four reverseworded items in the BFNE form (.43). The reliability coefficients of the BFNE-II and the BFNE-S were .90 and .87 respectively.

\section{Conclusion}

In the CFA study performed for three forms of the scale, sufficient validity evidence for the BFNE form was not reached. Although there is adequate evidence for the BFNE-II form, the validity evidence for the BFNE$\mathrm{S}$ form is psychometrically stronger. Based on these results, it can be said that the use of the tool with the BFNE-S form has valid and reliable properties in measuring the fear of negative evaluation of high school and university students. 


\section{Limitations and Future Directions}

The Brief Fear of Negative Evaluation Scale is a tool that can be used to measure social anxiety in research and practice (psychological counsellingclinical-diagnosis-evaluation). It is necessary to examine which of these three forms of the tool better discriminates between individuals with and without social anxiety. Furthermore, this study was conducted with high school and university students. Examining the validity and reliability of the tool with adults who are not involved in academic life will provide further information about the use of the tool with other groups.

\section{References:}

1. Ben, S. (2017). Relationship between social anxiety levels and body perception, fear of negative evaluation, social appearance anxiety of university students living in İstanbul (Unpublished master dissertation). Haliç University, İstanbul

2. Bilge, F. \& Kelecioğlu, H. (2008). Psychometric properties of The brief fear of negative evaluation scale: Turkish form, Eurasian Journal of Educational Research, 32, 21-38.

3. Carleton, R. N., Collimore, K. C. ve Asmundson, G. J. G. (2007). Social anxiety and fear of negative evaluation: Construct validity of the BFNE-II, Journal of Anxiety Disorders, 21, 131-141.

4. Carleton, R. N., Collimore, K. C., McCabe, R. E., \& Antony, M. M. (2011). Addressing revisions to the brief fear of negative evaluation scale: Measuring fear of negative evaluation across anxiety and mood disorders. Journal of Anxiety Disorders, 25, 822-828

5. Carleton, R. N., McCreary, D. R., Norton, P. J., \& Asmundson, G. J. G. (2006). Brief fear of negative evaluation scale-revised. Depression and Anxiety, 23, 297-303.

6. Collins, K. A., Westra, H. A., Dozois, D. J. A., \& Stewart, S. H. (2005). The validity of the brief version of the fear of negative evaluation scale. Journal of Anxiety Disorders, 19, 345-359

7. Çetin, B., Doğan, T. and Sapmaz, F. (2010). The Turkish adaptation of The brief negative evaluation scale: The validity and reliability study, Education and Science, 35(156) 205-216.

8. Çetinkaya-Yıldız, E., \& Toprak, E. (2016). An investigation of counselor candidates' empathic tendency, shyness, fear of negative evaluation and social skills in relation to varied variables. Mediterranean Journal of Humanities, 6(2), 513-530.

9. Doğan, T., \& Totan, T. (2010). The study of validity and reliability of the Turkish version of The fear of positive evaluation scale. Akademik Bakış Dergisi, 22, 1-20. 
10. Duke, D., Krishnan, M., Faith, M., \& Storch, E. A. (2006). The psychometric properties of The brief fear of negative evaluation scale. Journal of Anxiety Disorders, 20,807-817.

11. Irmak, M. (2015). An examination of secondary level students' fear of negative evaluation and their attitudes towards learning (Unpublished master dissertation). Erciyes University, Kayseri

12. Karademir, T. (2011). The assessment of parental attitudes towards the fear of negative evaluation of hearing and hearing impaired soccer players. (Unpublished doctoral dissertation), Firat University, Elazı $\breve{g}$.

13. Koydemir, S. and Demir, A. (2007). Psychometric properties of The brief fear of negative evaluation scale in a Turkish sample, Psychological Reports, 100, 883-893.

14. Le Blanc, A. L., Bruce, L. C., Heimberg, R. G., Hope, D. A., Blanco, C., Schneier, F. R., \& Liebowitz, M. R. (2014). Evaluation of the psychometric properties of two short forms of The social interaction anxiety scale and The social phobia scale. Assessment, 21(3), 312-323.

15. Leary, M, R. (1983). A brief version of The fear of negative evaluation scale, Personality and Social Psychology Bulletin, 9, 371-375.

16. Levinson, C. A., Rodebaugh, T. L., White, E. K., Menatti, A. R., Weeks, J. W., Iacovino, J. M., \& Warren, C. S. (2013). Social appearance anxiety, perfectionism, and fear of negative evaluation. Distinct or shared risk factors for social anxiety and eating disorders? Appetite, 67, 125-133.

17. Liu, L. \& Lowe, P. A. (2016). Examination of The brief fear of negative evaluation scale-version 2 and The brief fear of negative evaluation scale-straightforward items factor structure in a sample of U.S. college students, Canadian Journal of School Psychology, 31 (2), $122-138$

18. Menatti, A. R., DeBoer, L. B. H., Weeks, J. W., \& Heimberg, R. G. (2015). Social anxiety and associations with eating psychopathology: Mediating effects of fears of evaluation. Body Image, 14, 20-28.

19. Ömür, Y. E., Aydın, R., \& Argon, T. (2014). Relationship between prospective teachers' fear of negative evaluation and their academic dishonesty tendencies. Journal of Education and Humanities: Theory and Practice, (9), 131-149.

20. Rodebaugh, T. L., Woods, C. M., \& Heimberg, R. G. (2007). The reverse of social anxiety is not always the opposite: the reverse-scored items of the social interaction anxiety scale do not belong. Behavior Therapy, 38, 192-206.

21. Rodebaugh, T. L., Woods, C. M., Thissen, D. M., Heimberg, R. G., Chambless, D. L., \& Rapee, R. M. (2004). More information from 
fewer questions: the factor structure and item properties of the original and brief fear of negative evaluation scale. Psychological Assessment, $16,169-181$

22. Seçer, İ., Halmatov, S., \& Gençdoğan, B. (2013). Emotional reactivity scale adaptation to Turkish: reliability and validity study. Sakarya University Journal of Education, 3(1), 77-89.

23. Sedighimornani, N., Rimes, K., Verplanken, B., \& Gauntlett-Gilbert, J. (2019). Acceptance of shame and embarrassment: Scale development and initial findings in a clinical sample. Journal of Contextual Behavioral Science, 12, 13-21.

24. Stopa, L. \& Clark, D. M. (2001). Social phobia: Comments on the viability and validity of an analogue research strategy and British norms for the fear of negative evaluation questionnaire. Behavioural and Cognitive Psychotherapy. 29 (4), 423-430.

25. Turner, S. M., McCanna, M. \& Beidel, D. C. (1987). Validity of the social avoidance and distress and fear of negative evaluation scales. Behaviour Research and Therapy, 25,113-115.

26. Ümmet D., Çağlar, A., İme, Y. \& Akyıl, Yusuf. (2018, October 25-27) The Analysis of the Role of Emotional Autonomy and the Fear of Negative Evaluation on Adolescent's Psychological Resilience [Conference presentation]. XX. International Congress on Psychological Counseling and Guidance, Samsun, Türkiye

27. Villarosa-Hurlocker, M. C., Whitley, R. B., Capron, D. W., \& Madson, M. B. (2018). Thinking while drinking: Fear of negative evaluation predicts drinking behaviors of students with social anxiety. Addictive behaviors, 78, 160-165.

28. Watson, D., \& Friend, R. (1969). Measurement of social-evaluative anxiety. Journal of Consulting and Clinical Psychology, 33, 448-457

29. Weeks, J. W., \& Howell, A. N. (2012). The bivalent fear of evaluation model of social anxiety: Further integrating findings on fears of positive and negative evaluation. Cognitive Behaviour Therapy, 41(2), 83-95.

30. Weeks, J. W., Heimberg, R. G., Fresco, D. M., Hart, T. A., Turk, C. L., Schneier, F. R., \& Liebowitz, M. R. (2005). Empirical validation and psychometric evaluation of the brief fear of negative evaluation scale in patients with social anxiety disorder. Psychological Assessment, 17, 179-190

31. Yap, K., Gibbs, A. L., Francis, A. J., \& Schuster, S. E. (2016). Testing the bivalent fear of evaluation model of social anxiety: The relationship between fear of positive evaluation, social anxiety, and perfectionism. Cognitive behaviour therapy, 45(2), 136-149. 